\title{
A Four-Wheel Steer-by-Wire Control Method Based on the Guaranteed Cost Control Theory
}

\author{
T.T. Zhang, T. Zhou \\ College of Vehicle and Energy \\ Yanshan University \\ China
}

\begin{abstract}
Based on front wheel steering vehicles, the ideal yaw rate model was constructed. Four-wheel $(4 \mathrm{~W})$ steer-by-wire control model of two-degree-of-freedom (DOF) was taken as the study object, according to characteristics of vehicle constant speed and steady-state conditions, the zero sideslip angle and idea yaw rate were regarded as conditions to solve $4 \mathrm{~W}$ steer-by-wire feed-forward controller. Taking vehicle mass, moment of inertia and tire cornering stiffness as control model perturbation items, the guaranteed cost control theory was adopted to design feedback controller. For simulation tests, the input method of angle step was used to evaluate performances of the system model tracking ideal states. The track of snake lane was chosen to make real vehicle road tests. The results show that controllers have a good tracking accuracy for ideal states of vehicle.
\end{abstract}

Keywords-4W steer-by-wire; feed-forward control; guaranteed cost control; handling stability

\section{INTRODUCTION}

The $4 \mathrm{~W}$ steering technology allows rear wheels to involve in the process of vehicle steering and control vehicle lateral movement, which improves vehicle handling and stability characteristics. The domestic and foreign scholars, such as Lai and Huang et al [1-4] have conducted more in-depth study, generally adopt the following methods: Front and rear steering angles are proportional; Dynamic compensation control, this method is mainly a steering angle and steering torque dynamic compensation; The proportion control of front wheel steering angle which combines with the proportion control of yaw rate et al. But the above methods do not solve the problem that the zero sideslip angle as the target easily leads to vehicle under steer, increasing the burden of drivers. Marino et al [5] found that front and rear wheels of $4 \mathrm{~W}$ steer-by-wire can realize active steering, further improve vehicle handling stability and comfort. Tian et al [6] designed $4 \mathrm{~W}$ steer-by-wire control strategy respectively with feed-forward and feed-back controller, but the designed controllers were still according to mode of $4 \mathrm{~W}$ steering controller. Based on sliding mode control theory, Zheng et al [7] adopted model tracking to design the controller, but only with state feed-back without feed-forward controller, which was not conducive to improve response speed and tracking accuracy. Yu et al [8] adopted the proportional control and yaw rate feed-back control, but could not realize the ideal yaw rate tracking.

The ideal yaw rate model was constructed based on front wheel steering vehicles. Taking the $4 \mathrm{~W}$ steer-by-wire control model of 2 DOF as the study object, according to characteristics of vehicle constant speed and steady-state conditions, the zero sideslip angle and idea yaw rate were regarded as conditions to solve $4 \mathrm{~W}$ steer-by-wire feed-forward controller. Taking the vehicle mass, moment of inertia and tire cornering stiffness as control model perturbation items, the guaranteed cost control theory was adopted to design the feedback controller. For simulation tests, the input method of angle step was used to evaluate performances of the system model tracking ideal states. The track of snake lane was chosen to make real vehicle road tests. The results show that controllers have a good tracking accuracy for ideal states of vehicle.

\section{OBTAINING THE IDEAL YAW RATE}

An ideal 4W steering control should make yaw rate gain consistent with the one of front wheel steering vehicle. To ensure that the feeling of driving does not change greatly, the ideal yaw rate is calculated based on 2 DOF dynamics model of front-wheel steering vehicle. The function of ideal yaw rate includes two aspects: First, it can be used as a constraint when the feed-forward controller is designed; Second, it can be used as an ideal state of the feed-back control. At the condition of low frequency steering angle input and steady response, the yaw rate and steering angle can be simplified to the first order link:

$$
\frac{r^{*}(s)}{\delta(s)}=\frac{G_{r}}{1+T_{r} s}
$$

Where, $G_{r}=\frac{u_{c}}{a+\frac{m a b u_{c}^{2}}{2 a L k_{2}}} ; T_{r}=\frac{I_{z} u_{c}}{2 k_{1} a L+m b u_{c}^{2}} ; \gamma^{*}$ is the ideal yaw rate; $G_{r}$ is the stability factor; $T_{r}$ is the delay time constant of the yaw rate; $\delta$ is the front-wheel steering angle of the driver input; $u_{c}$ is the speed; $m$ is the vehicle mass; $a, b$ are respectively the distance between the vehicle mass center and the front/rear axle; $I_{z}$ is the rotational inertia of the vehicle; $k_{1}$, $k_{2}$ are respectively the front and rear cornering stiffness; $L$ is the wheelbase.

\section{THE CONTROLlERS DESIGN}

\section{A. Building the Vehicle Control Model}

The 4W steering electric vehicle developed by our research group is taken as the research object. The chassis assembly consists of power system, steering system, suspension system and brake system. Electrical assembly consists of power system, drive control system, steering control system, 
measurement and control system, consoles et al. The main parameters of vehicle are shown in table 1:

TABLE I. THE MAIN PARAMETERS OF THE VEHICLE.

\begin{tabular}{|c|c|c|c|c|c|}
\hline Parameters & $\begin{array}{c}L \\
(\mathbf{m m})\end{array}$ & $\begin{array}{c}\text { Tread } \\
B \\
(\mathbf{m m})\end{array}$ & $\begin{array}{l}\text { Front and } \\
\text { rear axle } \\
\text { ratio }\end{array}$ & $\begin{array}{c}a \\
(\mathbf{m m})\end{array}$ & $\begin{array}{c}b \\
(\mathbf{m m})\end{array}$ \\
\hline Value & 1650 & 1000 & $54 / 46$ & 798 & 798 \\
\hline Parameters & $\begin{array}{c}k_{1}, k_{2} \\
(\mathrm{~N} / \mathrm{rad})\end{array}$ & $\begin{array}{c}V_{\max } \\
\left(\mathrm{km} \cdot \mathrm{h}^{-}\right. \\
\left.{ }^{1}\right)\end{array}$ & $\begin{array}{c}I_{\mathrm{z}} \\
\left(\mathrm{kg} \cdot \mathrm{m}^{2}\right)\end{array}$ & $\begin{array}{c}m \\
(\mathrm{~kg})\end{array}$ & \\
\hline Value & 36000 & 50 & 365 & 495 & \\
\hline
\end{tabular}

The 4W steer-by-wire vehicle dynamics control model is shown as eqn (2):

$$
\dot{x}(t)=A x(t)+B u(t)
$$

Where, $A=\left[\begin{array}{cc}-\frac{K_{1}+K_{2}}{m u_{c}} & \frac{b K_{2}-a K_{1}}{m u_{c}^{2}}-1 \\ \frac{b K_{2}-a K_{1}}{I_{z}} & -\frac{a^{2} K_{1}+b^{2} K_{2}}{I_{z} u_{c}}\end{array}\right] ; \quad B=\left[\begin{array}{cc}\frac{K_{1}}{m u_{c}} & \frac{K_{2}}{m u_{c}} \\ \frac{a K_{1}}{I_{z}} & -\frac{b K_{2}}{I_{z}}\end{array}\right]$; $u=\left[\begin{array}{l}\delta_{f} \\ \delta_{r}\end{array}\right] ; x=\left[\begin{array}{l}\beta \\ \gamma\end{array}\right]$.

Where, $\beta$ is the side silp angle; $\gamma$ is the yaw rate; $\delta_{f}$ is the front wheel steering angle; $\delta_{r}$ is the rear wheel steering angle.

\section{B. The Feed-Forward Controller Design}

When the vehicle is in uniform steady state, $\dot{\beta}=0$, $\dot{\gamma}=0$, according to eqn (2), making $\beta=0$, eqn (3) can be obtained:

$$
\left\{\begin{array}{l}
\delta_{f}=\frac{\alpha_{2} \gamma^{*}}{\alpha_{3}\left(\alpha_{1}+\alpha_{2}\right)} \\
\delta_{r}=\frac{-\alpha_{1} \gamma^{*}}{\alpha_{3}\left(\alpha_{1}+\alpha_{2}\right)}
\end{array}\right.
$$

Eqn (3) is the feed-forward controller, which can ensure the sideslip angle is zero when vehicle steering, but also can ensure the yaw rate is equal to the ideal.

\section{The Feed-Back Controller Design}

As the control model parameters variation and external disturbance, there is a certain perturbation for control model; the control model is shown as eqn (4):

$$
\dot{x}_{1}(t)=(A+\Delta A) x_{1}(t)+(B+\Delta B) u
$$

Where, $x_{1}(t)=\left[\begin{array}{ll}e_{\beta} & e_{\gamma}\end{array}\right]^{\prime} ; \quad e_{\beta}=\beta^{*}-\beta ; \quad e_{\gamma}=\gamma^{*}-\gamma ; \quad \beta^{*}=0 ;$ Parameter matrix $A, B$ are the same with eqn (2); uncertain matrix function $\Delta A$ and $\Delta B$ are the uncertainty of model parameter. The uncertainty of the considering model parameter is norm bounded, $\Delta A$ and $\Delta B$ can be expressed as:

$$
\left[\begin{array}{ll}
\Delta A & \Delta B
\end{array}\right]=D F(t)\left[\begin{array}{ll}
E_{1} & E_{2}
\end{array}\right]
$$

Where, $D, E_{1}, E_{2}$ are known constant matrixs, $F(t)$ is unknown, $F(t) \in R^{i * j}$ is an unknown and time varying matrix, meeting the inequality:

$$
F^{T}(t) F(t) \leq I
$$

Definition of quadratic performance index is as follows:

$$
J=\int_{0}^{\infty}\left[X_{1}^{T}(t) Q x_{1}(t)+u^{T}(t) R u(t)\right] d t
$$

For eqn (5), if there is a state feed-back control law $u^{*}(t)$ and a positive number $J^{*}$, which make the closedloop system asymptotically stable and the performance indexes meet the inequality $J \leq J^{*}$ for all allow uncertainty, then $J^{*}$ is called the upper bound of performance of uncertain systems, which is called by $\mathrm{Yu}$ [9] a guaranteed cost control law of uncertain systems.

Theorem 1: for a given system (eqn (4)) and performance index, if the following optimization problems:

$$
\min _{\varepsilon, W, X, M} \operatorname{Trace}(M)
$$

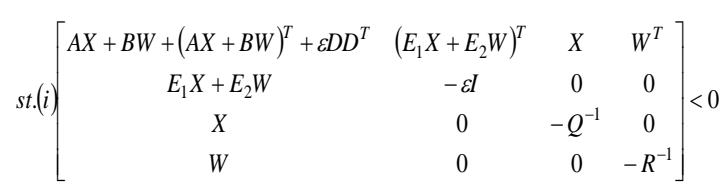

$$
\text { (ii) }\left[\begin{array}{cc}
M & I \\
I & X
\end{array}\right]>0
$$

Which have a solution $(\tilde{\varepsilon}, \tilde{W}, \tilde{X}, \tilde{M})$, so $u^{*}(t)=\tilde{W} \tilde{X}^{-1} X_{1}(t)$ is the optimal state feed-back guaranteed cost control law of the system. The problem is a convex optimization problem with linear matrix inequality (LMI) constraints, which can be solved by the mincx solver in LMI toolbox.

The model parameter perturbations including vehicle mass, moment of inertia, speed and tire cornering stiffness, the vehicle mass and moment of inertia have $\pm 15 \%$ floating, the speed has $\pm 5 \mathrm{~km} / \mathrm{h}$ floating, the tire cornering stiffness has $\pm 15 \%$ floating, the values of $Q$ and $R$ are:

$$
Q=\left[\begin{array}{ll}
4 & 0 \\
0 & 2
\end{array}\right], R=\left[\begin{array}{ll}
2 & 0 \\
0 & 1
\end{array}\right]
$$

According to theorem 1, the feed-back controller output can be obtained as: 


$$
u(t)=\tilde{W} \tilde{X}^{-1} x_{1}(t)=\left[\begin{array}{ll}
K_{11} & K_{12} \\
K_{21} & K_{22}
\end{array}\right] x_{1}(t)
$$

\section{The 4w Steering Vehicle Simulation Tests ANALYSIS}

Selecting two kinds of commonly used four wheel steering control methods, compared with the above designed controller. One is a dynamic compensation control method such as eqn (7); the other is the front wheel steering angle proportional control method such as eqn (8).

$$
\begin{gathered}
\delta_{r}=-c_{1} \delta_{f}+c_{2} \gamma u_{c} \\
\delta_{r}=k_{r f} \delta_{f}
\end{gathered}
$$

Where: $\quad c_{1}=1.0 ; \quad c_{2}=\frac{m b}{k_{1} L}+\frac{m a}{K_{2} L} ; \quad K_{f f}=\frac{-b+\frac{m a}{2 L K_{2}} u_{c}^{2}}{a+\frac{m b}{2 L k_{1}} u_{c}^{2}}$

Figure. 1 and Figure. 2 are the contrast of three kinds controllers: angle step input response with the $u_{\mathrm{c}}=30 \mathrm{~m} / \mathrm{s}, \delta=$ $0.03 \mathrm{rad}$, simulation tests of eqn (7) controller and simulation tests of eqn (8). Figure. 1 is response curves only used a feedforward controller designed in this paper, Figure. 2 is the response curves with the feed-forward and feed-back controllers with the maximum perturbation.
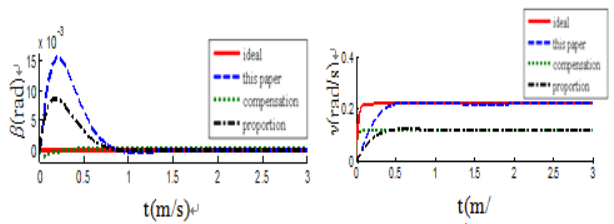

(a) the contrast of the sideslip angles. (b) the contrast of the yaw rate FIGURE I. THE CONTRAST OF THE FEED-FORWARD CONTROLLER SIMULATION.

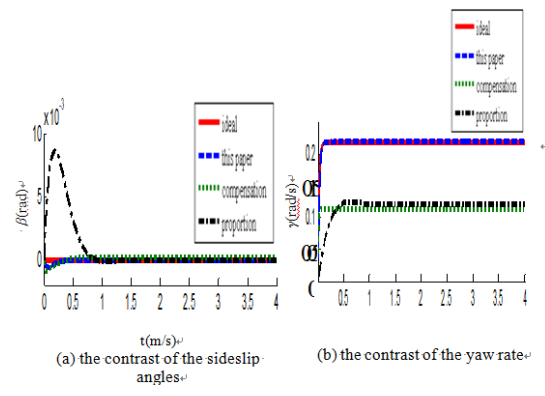

FIGURE II. THE CONTRAST OF THE FEED-FORWARD AND FEEDBACK CONTROLLERS SIMULATION.

As we can see from Figure. 1, system can better track $\gamma^{*}$ under the effect of the feed-forward controller, but the response speed is slower than that of the dynamic compensation method; although $\beta$ is the largest, but its value is very small, close to zero, so the comprehensive control under the effect of feed-forward controller is better than other controllers. As we can see from Figure. 2, under the effect of feed-forward and feed-back controllers, the overshoot and the steady state value $\beta$ are the minimum, the response speed $\gamma$ increases greatly and coincides with $\gamma^{*}$, which shows that the designed controllers in this paper can better track speed, have good precision and robustness for system perturbation.

\section{REAL VEHICLE TESTS}

The $4 \mathrm{~W}$ steer-by-wire control program is compiled in the VC6.0 development environment, the output steering angles of driver model under the serpentine line simulation condition are written into the control program. Figure. 3 is the experimental model car and Figure. 4 is the serpentine line tests.

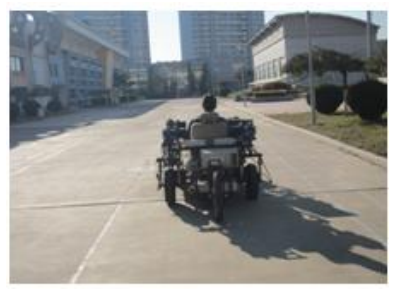

FIGURE III. THE TESTS SCENE.

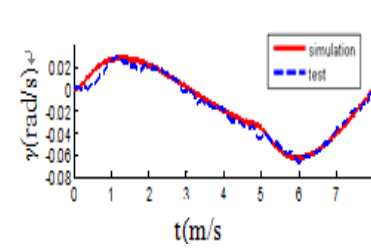

(a) the contrast of the yaw.

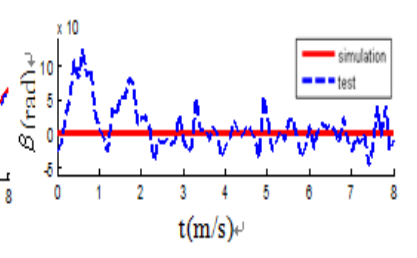

(b) the contrast of the sideslip angles
FIGURE IV. THE SERPENTINE LINE TESTS.

As shown in Figure. 4, the yaw rate obtained by real tests can faster and better track simulation values. The yaw rate, lateral acceleration, the sideslip angles measured by real tests have small error compared with the ones of simulation tests, so the $4 \mathrm{~W}$ steering performance designed in this paper is good.

\section{CONCLUSIONS}

(1) The zero sideslip angle and ideal yaw rate are regarded as conditions to solve the $4 \mathrm{~W}$ steer-by-wire feed-forward controller. Only under the effect of feed-forward controller, vehicle can achieve good control effect. The ideal yaw rate is needed to solve the feed-forward controller which is easy to apply in engineering.

(2) To select the perturbation of control model, the guaranteed cost control theory is used to design the feed-back controller. The controller has stronger adaptability for system perturbation and can improve the robustness.

(3) The tests show that the controllers designed in this paper have better tracking accuracy for the ideal system states than the traditional $4 \mathrm{~W}$ steering controllers. At the same time, the driver busy degree is less than the one of the traditional $4 \mathrm{~W}$ steering controllers.

\section{ACKNOWLEDGEMENTS}

This work is financially supported by the important program of science and technology research. 


\section{REFERENCES}

[1] Lai, F. \& Huang, C.Q., A Study on the variable-gain tracking control for the four-wheel steering system of vehicles. Automotive Engineering, 34(6), pp. 517-522, 2012.

[2] Song, Y., Chen, W.W., \& Chen, L.Q., A study on the hybrid control of four-wheel-steering vehicle based on yaw rate feedback and neural network adaptive control. Automotive Engineering, 35(1), pp. 66-71, 2013.

[3] Ren, D.B., Cui, S.M. \& Zhang, G.Z., Analysis of lane keeping control and steady state performance for four-wheel steering vehicle. Journal of Highway and Transportation Research and Development, 26(s1), pp. 7982, 2009.

[4] Wang, S.F., Zhang, J.Y. \& Li, H.S., Analysis of vehicle performance with multi-axle steering at different steering modes. Journal of Highway and Transportation Research and Development, 25(12), pp. 184-187, 2008.

[5] Marino, R., Scalzi, S. \& Cinili, F., Nonlinear PI front and rear steering control in four-wheel steering vehicles. Vehicle System Dynamics, 45(12), pp. 1149-1168, 2007.

[6] Tian, C.G., Zong, C.F. \& He, L., Control strategy of 4W steer-by-wire system. Journal of Jilin University (Engineering and Technology Edition), 40(5), pp. 1177-1182, 2010.

[7] Zheng, K.F., Chen, S.Z. \& Wang, Y., Four-wheel steering with total sliding mode control based on control-by-wire. Journal of Southeast University (Natural Science Edition), 43(2), pp. 334-339, 2013.

[8] Yu, L.Y., Lin, Y. \& Shi, G.B., Research on steering control strategy of four-wheel-steer-by-wire system. Computer Simulation, 25(4), pp. 278281, 2008.

[9] Yu, L., Robust control-linear matrix inequality processing method. Tsinghua university publishing house. 2002. 